\title{
An Interdisciplinary Reading of Mathematics
}

Yogendra Prasad Shah*

\begin{abstract}
This research paper recapitulates the history of mathematics, which occupies itself describing processes of growth and development, whereas philosophy of mathematics is concerned with questions of justification. Both play an essential role within the educational context. However, there is a problem because genuine historical studies necessitate ever-greater particularity whereas mathematics and philosophy require generality and abstraction. The paper offers some methodological reflections about these matters together with two case studies from nineteenth century history of arithmetic and integration theory, respectively, which try to strike a balance between the directly opposed requirements.
\end{abstract}

Keywords: Axiomatization, Arithmetic, Integration, Lebesgue, Grassmann, Mathematical and Generalization.

\section{Introduction}

This paper demonstrates the history of mathematics. Math is the science that deals with the logic of shape, quantity, and arrangement. Since the beginning of recorded history, mathematic discovery has been at the forefront of every civilized society, and in use in even the most primitive of cultures. The needs of math arose based on the wants of society. The more complex a society, the more complex the mathematical needs. Primitive tribes needed little more than the ability to count, but also believed on math to calculate the position of the sun and the physics of hunting. Several civilizations including China, India, Egypt, Central America and Mesopotamia contributed to mathematics. The Sumerians were the first people to develop a counting system. Mathematicians developed arithmetic, which includes basic operations, multiplication, fractions, and square roots. The Sumerians' system passed through the Akkadian Empire to the Babylonians around 300 B.C. Six hundred years later, in America, the Mayans developed elaborate calendar systems and were skilled astronomers. Since then, the concept of zero was developed.

When civilizations developed, mathematicians began to work with geometry, which computes areas and volumes to make angular measurements and has many sensible applications. Geometry is used in everything from home construction to fashion and interior design. Geometry went hand in hand with algebra, invented in the ninth century by a Persian

\footnotetext{
${ }^{*}$ Mr. Shah is a Lecturer of the Department of Mathematics, T.U., Patan Multiple Campus,Patan Dhoka.
} 
mathematician, Mohammed ibn-Musa al-Khowarizmi. He also developed quick methods for multiplying and diving numbers, which are known as algorithms - a corruption of his name.

Algebra offered civilizations a way to divide inheritances and allocate resources. The study of algebra meant mathematicians were solving linear equations and systems, as well as quadratics, and delving into positive and negative solutions. Mathematicians in ancient times also began to look at number theory. With origins in the construction of shape, number theory looks at figurate numbers, the characterization of numbers, and theorems.

The study of math within early civilizations was the building blocks for the math of the Greeks, who developed the model of abstract mathematics through geometry. Greece, with its unbelievable architecture and complex system of government, was the model of mathematic achievement until modern times. In addition to the Greek mathematicians, a number of Greeks made an indelible mark on the history of mathematics. Archimedes, Apollonius, Diophantus, Pappus, and Euclid all came from this era.

During this time, mathematicians began working with trigonometry. Computational in nature, trigonometry requires the measurement of angles and the computation of trigonometric functions, which include sine, cosine, tangent, and their reciprocals. Trigonometry relies on the synthetic geometry developed by Greek mathematicians like Euclid. For example, Ptolemy's theorem gives rules for the chords of the sum and difference of angles, which correspond to the sum and difference formulas for sines and cosines. In past cultures, trigonometry was applied to astronomy and the computation of angles in the celestial sphere.

After the fall of Rome, the Arabs, then the Europeans took on the development of mathematics. Fibonacci was one of the first European mathematicians, and was famous for his theories on arithmetic, algebra, and geometry. The Renaissance led to advances that included decimal fractions, logarithms, and projective geometry. Number theory was greatly expanded upon, and theories like probability and analytic geometry ushered in a new age of mathematics, with calculus at the forefront.

Pure mathematics is driven by abstract problems, rather than real world problems. Much of what's pursued by pure mathematicians can have their roots in concrete physical problems, but a deeper understanding of these phenomena brings about problems and technicalities. These abstract problems and technicalities are what pure mathematics attempts to solve, and these attempts have led to major discoveries for humankind, including the Universal Turing Machine, theorized by Alan Turing in 1937. The Universal Turing 
Machine, which began as an abstract idea, later laid the groundwork for the development of the modern computer. Pure mathematics is abstract and based in theory, and is thus not constrained by the limitations of the physical world.

As per one pure mathematician, pure mathematicians prove theorems, and applied mathematicians construct theories. Pure and applied are not mutually exclusive, but they are rooted in different areas of math and problem solving (Elaine, $2013: 16$ ).

\section{Interdisciplinary Approach to Mathematics}

One great problem of mathematics education is the seemingly static and infallible character of mathematical knowledge. Everything just is and thus means itself: $\mathrm{P}=\mathrm{P}$ ! This principle of identity lies at the heart of logic or exact science, and it is obviously directed against any historical or evolutionary concerns. P just means P! No psychological or philosophical consideration shall be able to add anything to the matter. Mathematics seems so immutable and absolute that the sociology and socio-cultural history of knowledge excluded it from their considerations since the very beginning. Within this context, it is claimed that mathematics has no history worth knowing. The newest state of the art of mathematics has taken up and reformulated in modern terms whatever appeared as worthwhile during its history. The history of mathematics is partly a dogma and partly gossip or small talk. The dogmatic attitude serves to exclude all alternative views of mathematics, whereas the small talk classifies them as off the record private ponderings.

Looking at mathematics in this way, however, leaves it as a set of completed works and finished theories that might sometimes reveal their secret beauty to the talented discoverer, but that could not be taught nor learned. Being a mere form of reality, or a reality suigeneris, it has nothing to do with human activities or emotions. Such a view does not allow, for example, the consideration of unresolved problems. This is not good, because great problems and programs of their investigation amount to the largest part of the "real" history of mathematics. In addition, it does not help to stimulate the spirit of creativity and truth in students. Yet the historical perspective on mathematics is essential to a spirit of truth and creativity. For instance, to perceive the changes in a thing certainly helps us to see it more clearly. The Continuity Principle has been the most important vehicle of mathematical generalization throughout the modern ages; it became dethroned only when a reductionist spirit of "rigor" took hold. One reason for this banishment or expulsion of the continuity principle and a motif for an illusionary search after a-historical absolute rigor was that this principle had not always been well understood in terms of the relevant practices. Continuity is something ideal; there is no perfect "uniformity of Nature", such that conceiving of 
change always requires constructive efforts and depends on epistemological views. This brings me to a different kind of caveat.

Historical considerations are often mere negative reactions to mathematical dogmatism and positivism, trying to use history as a means to debunk the assumption of a linear and logical development of mathematics as an illusion and sometimes meandering into abstract ideological criticisms. Mastery, Valéry says, presupposes that "one has the habit of thinking and combining directly from the means of activity, of imagining a work only within the limits of the means at hand, and never approaching a work from a topic or an imagined effect that is not linked to the means" (Valéry, $1960: 40$ ). The historical approach therefore cannot be justified by just pointing out "human" concerns or interests. And even the broadest socio-cultural approach to the history of mathematics has to model its principal categories so as to make them applicable to the mathematical and cognitive questions at hand. We should hence aim at a historiography of mathematics where the facts are being illuminated by epistemological and cultural reflection. Lakatos used to famously paraphrase Kant, "The history of mathematics, lacking the guidance of philosophy, is blind, while the philosophy of mathematics, turning its back on the most intriguing phenomena in the history of mathematics, is empty" (Lakatos, 1970 : 135). Differing from Lakatos, we do not believe, however, that a particular philosophy of mathematics could be "proven" right from history or that there is a completely "rational reconstruction" of the historical developments.

No historical study can resolve these controversies and it would not even be the purpose of such studies. Their purpose is first to describe and analyze attitudes, rather than justify them. Unlike philosophers or educators, historians are unlikely to be focusing upon justification. "Reality" is a culturally laden concept and is certainly more than a set of material facts or objects. Positivists were therefore suspicious of mathematics because the objects of mathematical study were not material. Mathematical positivists opted for formal definitions and rigorous proofs to escape such suspicions. We believe that it is this question that is addressed when Thom affirms, "the real problem which confronts mathematics teaching is not that of rigor, but the problem of the development of meaning, of the 'existence' of mathematical objects" (Thom, 1973 : 202). To tackle this problem, students should experience processes of generalization and abstraction, rather than being confronted with the abstract as such.

During the nineteenth century, there appeared a number of problems and certain transitions occurred, based on new theoretical ideas about the nature of mathematics and its objects. These developments - that may be cast in terms of a transformation of Euclidean 
axiomatics into set theory and formal axiomatics-made mathematics more abstract and less "explanatory". It seems a curious historical fact indeed that abstract pure mathematics arose not least from the necessities of large scale or distant communication, the irony being that its formal character simultaneously facilitates and impedes learning and communication. Learning difficulties result not least from the fact that the concept of "explanation" is central to our educational practices and aims, whereas modern science and mathematics do not provide explanations of anything in the sense desired. They are either too hypothetical and abstract or too instrumental and technical. Mathematics could not, however, be fruitfully organized and pursued at school or university as a primarily professional topic. Mathematical education, like other subjects, also must contribute to a common search for clarity on fundamental issues. It might be concluded therefore that axiomatics in the sense of Euclid, i.e. "logical reduction, analysis and organization of intelligible concepts and meaningful sentences, seems to remain an irreducible, fundamental tool of our thinking" (Casari, 1974 : 61). This does not mean that everybody would want to neither go back to the style and methods of Euclidean mathematics nor stimulate a mathematical fundamentalism that aims to reduce everything to elementary mathematics. Nevertheless, it does show that modern mathematics has been divided in its epistemology and methodology since the beginning of modernity.

The previous ontological developments concerning the nature of mathematics and mathematical objects were underpinned by epistemological changes whose understanding is important if we want to employ the history of mathematics for educational purposes. There was a shift from direct or constructive approaches to indirect and analytical ones and from instrumental reasoning to relational thinking.

Since the nineteenth century, the foundations of the mathematical edifice turned into mere hypotheses to be justified by their possible consequences. As Peirce put it, mathematics is concerned with ideal states of things. In this way, we systematize and analyze our thoughts. This process reflects on itself. Mathematics is in a sense metamathematics or meta-knowledge. Peirce himself, contrary to the majority of philosophers and mathematicians, however, did not endorse a completely analytical ideal of mathematics, but rather conceived of mathematical relations as establishing objective possibilities within a dynamical reality.

Even the analytic attitude brought by the modern axiomatic movement is an activity and as an activity, it requires "objects" to act on. There are always unexpected facts and things without an explanation (even in pure mathematics as (Chaitin, 1998 : 54) has emphasized, for example), contrary to Leibniz' principle of sufficient reason and in contrast 
to his belief that essence precedes existence. We should therefore not exaggerate the analytical attitude, as if one could foresee all the consequences of one's views or assumptions.

Mathematics can be better conceptualized as a recursive interaction between relational and operative forms of reasoning. Operative thinking and a functionalist perspective introduced by this operativity were what made possible the transition from the mathematics of Antiquity to the algebraic outlook beginning with Descartes. A complementary aspect of this process, which was equally indispensable, may be called relational thinking. Leibniz thought that truth is constituted by proof; Descartes believed in evidence and thought proof irrelevant to truth. Leibniz believed in relations and theories, Descartes in facts and in the instrumental aspects of problem solving. It is the relational thought system that allowed man to develop mathematics and science and that became dominant at the beginning of the nineteenth century, when algebra was transformed from a "language" into a science of structures. The operative conceptual schemata themselves, in a way, had to become an object of thinking. Algebra is meta-algebra, it is "algebra on algebra" as Sylvester (18141897 ) once remarked with particular reference to the algebra of determinants.

Number could have been turned into a language for all of mathematics already during the seventeenth century, but "arithmetization" became a program during the nineteenth century only after the algebraic perspective had sensitized mathematics to the structural view (Otte and Jahnke, 1981). There have therefore been two different trends in the foundational debate of mathematics during the nineteenth century, each of which emphasized nearly exclusively one of two fundamental cognitive operations: the search for similarities and relations or the drawing of definite distinctions. The arithmetizing program, being of the latter kind and based on rigor, searched to solve the foundational problems in a reductionistic manner, by defining all mathematical concepts in terms of some basic reified entities, ultimately the natural numbers. Complex numbers were for Cauchy nothing but pairs of real numbers. The axiomatic movement tried to employ, so to say, a synthetic top-down strategy, solving the foundational problems of mathematics by extending and generalizing its relational structures and its rules of inference, thereby enlarging the applicability of its theories. These two views, the synthetical and the analytical, have always more or less made up the essence of mathematical activity (Otte and Panza, 1997 : 104). Mathematical activity thus occurs by a sort of recursive interaction between reification and generalization. Mathematical objects are but hypostatic abstractions. "Hypostatic abstraction" means that a thought becomes the object of another thought. This is very fundamental for modern mathematical thinking. Thurston describes it under the label of mental compression. Mathematics is amazingly 
compressible: you may struggle a long time, gradually to work through some process or idea from several approaches.

\section{Conclusion}

Mathematics is a methodical application of matter because the subject makes a human methodical or systematic. Mathematics makes our life orderly and prevents chaos. Certain qualities that are nurtured by mathematics are power of reasoning, creativity, abstract or spatial thinking, critical thinking, problem-solving ability and even effective communication skills. Indeed, mathematics has integral relations with all disciplines such as physics, chemistry, biology, social science, history, etc because all of them function in logical manners.

\section{References}

Casari, E. (1974). "Axiomatical and Set-theoretical Thinking". Synthese, 27, 49-62.

Chaitin, G. J. (1998). The Limits of Mathematics. Berlin Heidelberg New York: Springer.

Elaine,J., (2013). "What is Mathematics?" Live Science. https://www.livescience.com/38936mathematics.html

Lakatos, I. (1970). "Falsification and the Methodology of Science Research Programs". In Lakatos/Musgrave (Eds.), Criticism and the Growth of Knowledge (pp. 91-196). Cambridge UP.

Otte, M., \& Jahnke, N. (1981). "Origins of the Program of Arithmetization of Mathematics". In H. Bos et al. (Eds.), Social History of Nineteenth Century Mathematics (pp. 21-49). Boston: Birkhäuser.

Otte, M., \& Panza, M. (Eds.) (1997). "Analysis and Synthesis in Mathematics". Boston Studies in the Philosophy of Sciences, 196.Peirce, Ch. S.: CP = Collected Papers of Charles Sanders Peirce, Volumes I-VI, C. Hartshorne and P. Weiß(Eds.), Cambridge, MA (Harvard UP) 1931-1935, Volumes VII-VIII, A. W. Burks (Eds.), Cambridge, MA. 